University of Windsor

Scholarship at UWindsor

1978

\title{
Angular integrals and radial recurrence relations for two-electron matrix elements in Hylleraas coordinates
}

Gordon W. F. Drake

University of Windsor

Follow this and additional works at: https://scholar.uwindsor.ca/physicspub

Part of the Physics Commons

\section{Recommended Citation}

Drake, Gordon W. F.. (1978). Angular integrals and radial recurrence relations for two-electron matrix elements in Hylleraas coordinates. Physical Review A, 18 (3), 820-826.

https://scholar.uwindsor.ca/physicspub/102

This Article is brought to you for free and open access by the Department of Physics at Scholarship at UWindsor. It has been accepted for inclusion in Physics Publications by an authorized administrator of Scholarship at UWindsor. For more information, please contact scholarship@uwindsor.ca. 


\title{
Angular integrals and radial recurrence relations for two-electron matrix elements in Hylleraas coordinates
}

\author{
G. W. F. Drake \\ Department of Physics, University of Windsor, Windsor, Ontario, Canada N9B $3 P 4$ \\ (Received 10 May 1978)
}

General formulas are obtained for the reduction of a wide class of two-electron matrix elements in Hylleraas coordinates to finite sums of radial integrals for states of arbitrary angular momentum. Multipole transition integrals and the Breit interaction are treated as special cases. A number of recurrence relations are derived for radial integrals containing $P_{L}(\cos \theta)$ (where $\cos \theta=\hat{r}_{1} \cdot \hat{r}_{2}$ ) in terms of radial integrals containing lower-order Legendre polynomials. The results are well suited to computer implementation.

\section{INTRODUCTION}

A large number of highly accurate variational calculations have now been done for the energies and other atomic properties of two-electron systems in terms of the well-known $r_{1}, r_{2}, r(r$ $\left.=\left|\vec{r}_{1}-\vec{r}_{2}\right|\right)$ Hylleraas coordinates. This work has dealt primarily with $S$ and $P$ states. However, for matrix elements involving states of high angular momentum, the evaluation of angular integrals and the reduction to radial integrals can become very laborious. The problem is complicated by the fact that if $r_{1}, r_{2}$, and $r$ are regarded as the independent variables in the radial integration, then only one of $\hat{r}_{1}$ and $\hat{r}_{2}$ may be treated as independent in the angular integration. Of the six independent variables, the three independent angles are the ones which determine the orientation in space of the triangle formed by $\overrightarrow{\mathrm{r}}_{1}, \overrightarrow{\mathrm{r}}_{2}$, and $\overrightarrow{\mathrm{r}}$.

The reduction of matrix elements to radial integrals over $r_{1}, r_{2}$, and $r$ has been studied by several author $\mathrm{s}^{1,2}$ for a number of special cases, but few results of a general nature have been published. The only previous work appears to be a general reduction to radial integrals for the overlap matrix element in terms of uncoupled angular momentum eigenfunctions by Calais and Löwdin. ${ }^{3}$ In this paper we collect together a number of general angular integration formulas for the reduction of the matrix elements of a wide class of operators to radial integrals. The wave functions are assumed to be vector coupled eigenfunctions of the total angular momentum operator $\overrightarrow{\mathrm{L}}=\overrightarrow{\mathrm{I}_{1}}+\overrightarrow{\mathrm{I}_{2}}$. Recurrence relations for the associated radial integrals are given to complete the efficient and automatic evaluation of matrix elements for states of arbitrary angular momentum. The recurrence relations derived by $\mathrm{Sack}^{4}$ for the coefficients in the Legendre polynomial expansion of functions of $r$ emerge as a special case of the present analysis.

In particular, we consider the general problem of evaluating integrals of the form

$$
\begin{aligned}
I=\iint & d \overrightarrow{\mathrm{r}}_{1} d \overrightarrow{\mathrm{r}}_{2} R^{\prime} Y_{l_{1}^{\prime} l_{2} L^{\prime}}^{M^{o}}\left(\hat{r}_{1}, \hat{r}_{2}\right) * \\
& \times T_{k_{1} k_{2} K^{\circ}}^{\odot} Y_{l_{1} l_{2} L}^{M}\left(\hat{r}_{1}, \hat{r}_{2}\right) R,
\end{aligned}
$$

where $Y_{l^{\circ} l^{\circ} L^{\prime}}^{M^{\prime}}, T_{k_{1} k_{2} K}^{Q}$, and $Y_{l_{1} l_{2} L}^{M}$ all denote vector coupled products of spherical harmonics of the form

$$
\begin{aligned}
& Y_{l_{1} l_{2} L}^{M}\left(\hat{r}_{1}, \hat{r}_{2}\right)=\sum_{m m}\left\langle l_{1} l_{2} m_{1} m_{2} \mid L M\right\rangle \\
& \times Y_{l_{1}}^{m_{1}}\left(\hat{r}_{1}\right) Y_{l_{2}}^{m_{2}}\left(\hat{r}_{2}\right) \\
& T_{k_{1} k_{2} K}^{Q}\left(\hat{r}_{1}, \hat{r}_{2}\right)=\sum_{\mu_{1} \mu_{2}}\left\langle k_{1} k_{2} \mu_{1} \mu_{2} \mid K Q\right\rangle \\
& \times Y_{k_{1}}^{\mu_{1}}\left(\hat{r}_{1}\right) Y_{k_{2}}^{\mu_{2}}\left(\hat{r}_{2}\right),
\end{aligned}
$$

and $R=R\left(r_{1}, r_{2}, r\right)$ denotes the correlated radial part of the function. We also consider integrals where $T_{K}^{Q}$ is constructed from the components of $\overrightarrow{\mathrm{p}}_{1}$ and/or $\overrightarrow{\mathrm{p}}_{2}$ instead of the components of $\overrightarrow{\mathrm{r}}_{1}$ and $\overrightarrow{\mathrm{r}}_{2}$. The results to follow apply whenever the integrals exist.

\section{REDUCTION TO RADIAL INTEGRALS}

Let us start by first evaluating the primitive integral

$$
\begin{aligned}
I\left(l_{1} m_{1}, l_{2} m_{2} ; R^{\prime} R\right)=\iint & d \overrightarrow{\mathrm{r}}_{1} d \overrightarrow{\mathrm{r}}_{2} R^{\prime} Y_{l_{1}}^{m_{1}\left(\hat{r}_{1}\right)^{*}} \\
& \times Y_{l_{2}}^{m_{2}}\left(\hat{r}_{2}\right) R .
\end{aligned}
$$

The radial integrals are conveniently done by integrating explicitly over the $r$ coordinate with the volume element ${ }^{5}$

$$
d \mathrm{r}_{1} d \mathrm{r}_{2}=r_{1} d r_{1} r_{2} d r_{2} r d r \sin \theta_{1} d \theta_{1} d \varphi_{1} d \chi
$$

where $\theta_{1}, \varphi_{1}$ are the polar angles of the vector $\overrightarrow{\mathbf{r}}_{1}$, and $\chi$ is the angle of rotation of the rigid triangle formed by $\vec{r}_{1}, \vec{r}_{2}$, and $\vec{r}$ about the $\vec{r}_{1}$ direction. In this coordinate system, $\theta_{2}$ and $\varphi_{2}$ are not indepen-

820 (C) 1978 The American Physical Society 
dent variables, but $Y_{l_{2}}^{m_{2}}\left(\theta_{2}, \varphi_{2}\right)$ in (4) can be expressed in terms of the independent variables by means of the rotation matrix relation ${ }^{6}$

$$
Y_{l_{2}}^{m_{2}}\left(\theta_{2}, \varphi_{2}\right)=\sum_{m} \mathbb{D}_{m_{2}, m}^{l_{2}}\left(\varphi_{1}, \theta_{1}, \chi\right)^{*} Y_{l_{2}}^{m}(\theta, \varphi),
$$

where $\theta, \varphi$ are the polar angles of $\vec{r}_{2}$ relative to $\overrightarrow{\mathbf{r}}_{1}$. Substitution of (6) into (4) and integration over $\theta_{1}, \varphi_{1}$, and $\chi$ then leads to the basic angular integral algorithm ${ }^{3}$

$$
I\left(l_{1} m_{1}, l_{2} m_{2} ; R^{\prime} R\right)=2 \pi \delta_{m_{1} m_{2}} \delta_{l_{1} l_{2}} I_{l_{1}}\left(R^{\prime} R\right),
$$

where

$$
\begin{aligned}
I_{l}\left(R^{\prime} R\right)=\int_{0}^{\infty} r_{1} d r_{1} \int_{0}^{\infty} & r_{2} d r_{2} \\
& \times \int_{\left|r_{1}-r_{2}\right|}^{r_{1}+r_{2}} r d r R^{\prime} R P_{l}(\cos \theta) .
\end{aligned}
$$

$P_{l}(\cos \theta)$ is a Legendre polynomial and $\cos \theta$ is a radial function defined by

$$
\cos \theta=\left(r_{1}^{2}+r_{2}^{2}-r^{2}\right) / 2 r_{1} r_{2}
$$

We return to the evaluation of the radial integrals in Sec. IV.

Let us consider now the full integral (1). Using standard angular-momentum coupling techniques together with the basic integral algorithm (7), the integral can be written in the form

$I=(-1)^{L^{\prime \prime}-M^{\prime}}\left(\begin{array}{ccc}L^{\prime} & K & L \\ -M^{\prime} & Q & M\end{array}\right) \sum_{\substack{\lambda_{1}, \lambda_{2} \\ \Lambda}} X_{\lambda_{1}, \lambda_{2}, \Lambda} D_{\lambda_{1}, \lambda_{2}, \Lambda} I_{\Lambda}\left(R^{\prime} R\right)$,

where $I_{\Lambda}\left(R^{\prime} R\right)$ is the radial integral (8) involving $P_{\Lambda}(\cos \theta)$,

$$
\begin{aligned}
X_{\lambda_{1}, \lambda_{2}, \Lambda}= & \frac{(-1)^{l_{1}^{\prime}+l_{2}^{\prime}+L^{\prime}+\Lambda}}{8 \pi}\left(\lambda_{1}, \lambda_{2}, \Lambda\right)\left(l_{1}, k_{1}, l_{1}^{\prime}, l_{2}, k_{2}, l_{2}^{\prime}, L, L^{\prime}, K\right)^{1 / 2} \\
& \times\left(\begin{array}{ccc}
l_{1} & k_{1} & \lambda_{1} \\
0 & 0 & 0
\end{array}\right)\left(\begin{array}{ccc}
l_{1}^{\prime} & \lambda_{1} & \Lambda \\
0 & 0 & 0
\end{array}\right)\left(\begin{array}{ccc}
l_{2} & k_{2} & \lambda_{2} \\
0 & 0 & 0
\end{array}\right)\left(\begin{array}{ccc}
l_{2}^{\prime} & \lambda_{2} & \Lambda \\
0 & 0 & 0
\end{array}\right),
\end{aligned}
$$

and $D_{\lambda_{1}, \lambda_{2}, \Lambda}$ is represented by the angular-momentum coupling graph ${ }^{6}$ shown in Fig. 1. We have used the notation $(\alpha, \beta, \ldots)=(2 \alpha+1)(2 \beta+1) \cdots$. The graph for $D_{\lambda_{1}, \lambda_{2}, \Lambda}$ can be separated across the
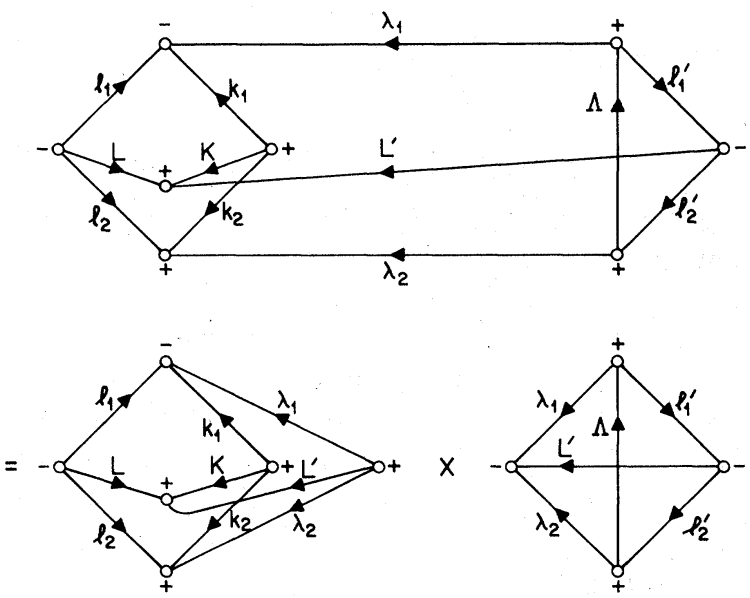

FIG. 1. Angular-momentum coupling graph from Eq. (10) showing the decomposition into the product of a $6-j$ and a 9-j symbol. All angular momenta are assumed to to be integers. Also $X_{\lambda_{1}, \lambda_{2}, \Lambda}$ defined by Eq. (11) vanishes unless each of the sums $l_{1}+k_{1}+\lambda_{1}, l_{1}^{\prime}+\lambda_{1}+\Lambda$, $l_{2}+k_{2}+\lambda_{2}$, and $l_{2}^{\prime}+\lambda_{2}+\Lambda$ is even. Each vertex represents a $3-j$ symbol as in Ref. 6 . $\lambda_{1}, \lambda_{2}, L^{\prime}$ lines into the product of a $6-j$ and a $9-j$ symbol as shown with the result

$$
D_{\lambda_{1}, \lambda_{2}, \Lambda}=\left\{\begin{array}{ccc}
L^{\prime} & l_{2}^{\prime} & l_{1}^{\prime} \\
\Lambda & \lambda_{1} & \lambda_{2}
\end{array}\right\}\left\{\begin{array}{lll}
l_{1} & l_{2} & L \\
k_{1} & k_{2} & K \\
\lambda_{1} & \lambda_{2} & L^{\prime}
\end{array}\right\} .
$$

The $9-j$ symbol reduces to a $6-j$ symbol if any index is zero. ${ }^{6}$ The above formulas thus express the integral (1) in the form

$$
I=\sum_{\Lambda} C_{\Lambda} I_{\Lambda}\left(R^{\prime} R\right)
$$

where the $C_{\Lambda}$ are the angular coefficients

$$
C_{\Lambda}=\sum_{\lambda_{1}, \lambda_{2}} C_{\lambda_{1}, \lambda_{2}, \Lambda}
$$

and

$$
C_{\lambda_{1}, \lambda_{2}, \Lambda}=(-1)^{L^{\prime}-M^{\prime}}\left(\begin{array}{ccc}
L^{\prime} & K & L \\
-M^{\prime} & Q & M
\end{array}\right) X_{\lambda_{1}, \lambda_{2}, \Lambda} D_{\lambda_{1}, \lambda_{2}, \Lambda} .
$$

Recurrence relations for the radial integrals $I_{\Lambda}\left(R^{\prime} R\right)$ are given in Sec. IV. 


\section{SPECIAL CASES}

\section{A. Scalar operators}

Consider matrix elements of operators of the form $T_{k, k, 0}^{0}\left(\hat{r}_{1}, \hat{r}_{2}\right)$. Using the definition (3), the simplest example is

$$
\overrightarrow{\mathrm{r}}_{1} \cdot \overrightarrow{\mathrm{r}}_{2}=-(4 \pi / \sqrt{3}) r_{1} r_{2} T_{1,1,0}^{0}\left(\hat{r}_{1}, \hat{r}_{2}\right)
$$

Since the $9-j$ symbol in (12) reduces to a $6-j$ symbol, the coefficients $C_{\lambda_{1}, \lambda_{2}, \Lambda}$ in (14) for the operator $T_{k, k, 0}^{0}\left(\hat{r}_{1}, \hat{r}_{2}\right)$ are

$$
\begin{aligned}
C_{\lambda_{1}, \lambda_{2}, \Lambda}\left(T_{k, k, 0}^{0}\right)= & \frac{(-1)^{\Lambda}}{8 \pi}\left(\lambda_{1}, \lambda_{2}, \Lambda\right)\left(k, l_{1}, l_{1}^{\prime}, l_{2}, l_{2}^{\prime}\right)^{1 / 2}\left(\begin{array}{ccc}
l_{1} & k & \lambda_{1} \\
0.0 & 0
\end{array}\right)\left(\begin{array}{ccc}
l_{1}^{\prime} & \lambda_{1} & \Lambda \\
0 & 0 & 0
\end{array}\right)\left(\begin{array}{lll}
l_{2} & k & \lambda_{2} \\
0 & 0 & 0
\end{array}\right)\left(\begin{array}{lll}
l_{2}^{\prime} & \lambda_{2} & \Lambda \\
0 & 0 & 0
\end{array}\right) \\
& \times\left\{\begin{array}{lll}
L & \lambda_{1} & \lambda_{2} \\
\Lambda & l_{2}^{\prime} & l_{1}^{\prime}
\end{array}\right\}\left\{\begin{array}{lll}
L & \lambda_{1} & \lambda_{2} \\
k & l_{2} & l_{1}
\end{array}\right\} \delta_{L, L^{\prime}} \delta_{M, M^{*}}
\end{aligned}
$$

The above form is useful in some applications because $\lambda_{1}$ and $\lambda_{2}$ label separately the various angular-momentum contributions which result when the operator is multiplied to the right into $Y_{l_{1}}^{m_{1}}\left(\hat{r}_{1}\right)$ and $Y_{l_{2}}^{m_{2}}\left(\hat{r}_{2}\right)$, respectively. For example, since

$$
\overrightarrow{\mathrm{r}} Y_{l}^{m}=-r\left(\frac{l+1}{2 l+1}\right)^{1 / 2} \overrightarrow{\mathrm{Y}}_{l, l+1}^{m}+r\left(\frac{l}{2 l+1}\right)^{1 / 2} \overrightarrow{\mathrm{Y}}_{l, l-1}^{m},
$$

where $\overrightarrow{\mathrm{Y}}_{l, \lambda}^{m}$ is a vector spherical harmonic, ${ }^{7}$ then $C_{\lambda_{1}, \lambda_{2}, \Lambda}\left(\overrightarrow{\mathbf{r}}_{1} \cdot \overrightarrow{\mathrm{r}}_{2}\right)$ is the contribution to $P_{\Lambda}(\cos \theta)$ from the term [cf. Eq. (2)],

$$
a_{1} a_{2} \sum_{m_{1}, m_{2}}\left\langle l_{1} l_{2} m_{1} m_{2} \mid L M\right\rangle r_{1} r_{2} \vec{Y}_{l_{1} \lambda_{1}}^{m_{1}}\left(\hat{r}_{1}\right) \cdot \vec{Y}_{l_{2} \lambda_{2}}^{m_{2}}\left(\hat{r}_{2}\right)
$$

with

$$
a_{i}= \begin{cases}-\left(\frac{l_{i}+1}{2 l_{i}+1}\right)^{1 / 2} & \text { if } \lambda_{i}=l_{i}+1 \\ \left(\frac{l_{i}}{2 l_{i}+1}\right)^{1 / 2} & \text { if } \lambda_{i}=l_{i}-1\end{cases}
$$

Similarly, $C_{\lambda_{1}, \lambda_{2}, \Lambda}\left(\overrightarrow{\mathbf{r}}_{1} \times \overrightarrow{\mathrm{r}}_{2}\right)$ is the corresponding contribution from $\overrightarrow{\mathrm{Y}}_{l_{1} \lambda_{1}}^{m_{1}}\left(\hat{\gamma}_{1}\right) \times \overrightarrow{\mathrm{Y}}_{l_{2} \lambda_{2}}^{m_{2}}\left(\hat{\gamma}_{2}\right)$ except that the general equation (15) must be used for the $C_{\lambda_{1}, \lambda_{2}, \Lambda}$ since the operator is now a tensor of rank 1. These results will be applied in Sec. III B.

For computational purposes, (17) can be expresed in a simpler form. After application of the Biedenharn-Elliot sum rule ${ }^{8}$ and two contractions of products of a $6-j$ symbol with two $3-j$ symbols ${ }^{9}$ the complete integral (13) can be written

$$
I\left(T_{k, k, 0}^{0}\right)=\frac{1}{4 \pi} \sum_{\Lambda} C_{\Lambda}(1) I_{\Lambda}\left[R^{\prime} R P_{k}(\cos \theta)\right],
$$

where

$$
\begin{aligned}
C_{\Lambda}(1)= & \frac{(-1)^{L+\Lambda}}{2}(2 \Lambda+1)\left(l_{1}, l_{1}^{\prime}, l_{2}, l_{2}^{\prime}\right)^{1 / 2} \\
& \times\left(\begin{array}{lll}
l_{1}^{\prime} & l_{1} & \Lambda \\
0 & 0 & 0
\end{array}\right)\left(\begin{array}{lll}
l_{2}^{\prime} & l_{2} & \Lambda \\
0 & 0 & 0
\end{array}\right)\left\{\begin{array}{lll}
L & l_{1} & l_{2} \\
\Lambda & l_{2}^{\prime} & l_{1}^{\prime}
\end{array}\right\}
\end{aligned}
$$

is the coefficient of $I_{\Lambda}\left(R^{\prime} R\right)$ in the overlap integral containing $4 \pi T_{0,0,0}^{0}\left(\hat{r}_{1}, \hat{r}_{2}\right)=1$, i.e.,

$$
I(1)=\sum_{\Lambda} C_{\Lambda}(1) I_{\Lambda}\left(R^{\prime} R\right)
$$

In (19), it is useful to use the rule for expanding products of Legendre polynomials as sums of Legendre polynomials so that

$$
I_{\Lambda}\left[R^{\prime} R P_{k}(\cos \theta)\right]=\sum_{K}(2 K+1)\left(\begin{array}{lll}
\Lambda & k & K \\
0 & 0 & 0
\end{array}\right)^{2} I_{K}\left(R^{\prime} R\right)
$$

B. Matrix elements involving the gradient operator and $\nabla^{2}$

The Breit interaction ${ }^{10}$ contains operators such as $\vec{r}_{1} \times \nabla_{2}, \vec{r}_{1} \cdot \nabla_{2}$, and $\nabla_{1} \cdot \nabla_{2}$. To reduce these operators to radial integrals, we separate the radial and angular parts of the gradient operators according to

$$
\begin{aligned}
& \nabla_{1}=\hat{r}_{1} \frac{\partial}{\partial r_{1}}+\frac{\left(\overrightarrow{\mathrm{r}}_{1}-\overrightarrow{\mathrm{r}}_{2}\right)}{r} \frac{\partial}{\partial r}+\nabla_{1}^{Y}, \\
& \nabla_{2}=\hat{r}_{2} \frac{\partial}{\partial r_{2}}+\frac{\left(\overrightarrow{\mathrm{r}}_{2}-\overrightarrow{\mathrm{r}}_{1}\right)}{r} \frac{\partial}{\partial r}+\nabla_{2}^{Y},
\end{aligned}
$$

where $\nabla^{Y}$ is understood to act only on spherical harmonics. Since the angular integrations for the radial parts can be performed by the methods already given in Secs. I and II we consider first only the $\nabla^{Y}$ part. The coefficients $C_{\lambda_{1}, \lambda_{2}, \Lambda}$ can be obtained from those for the closely related operators

$$
\begin{aligned}
& \left(\hat{r}_{1} \times \hat{r}_{2}\right)_{Q}=-(i 4 \pi \sqrt{2} / 3) T_{111}^{Q}\left(\hat{r}_{1}, \hat{r}_{2}\right) \\
& \hat{r}_{1} \cdot \hat{r}_{2}=(-4 \pi / \sqrt{3}) T_{110}^{0}\left(\hat{r}_{1}, \hat{r}_{2}\right)
\end{aligned}
$$

by noting that

$$
\begin{aligned}
\nabla Y_{l}^{m}= & -\left(\frac{l+1}{2 l+1}\right)^{1 / 2}\left(\frac{-l}{r}\right) \overrightarrow{\mathrm{Y}}_{l, l+1}^{m} \\
& +\left(\frac{l}{2 l+1}\right)^{1 / 2}\left(\frac{l+1}{r}\right) \overrightarrow{\mathrm{Y}}_{l, l-1}^{m} .
\end{aligned}
$$

Comparing (26) with (18) shows that 


$$
C_{\lambda_{1}, \lambda_{2}, \Lambda}\left(\hat{r}_{1}, \nabla_{2}^{Y}\right)=\frac{b_{\lambda_{2}}}{r_{2}} C_{\lambda_{1}, \lambda_{2}, \Lambda}\left(\hat{r}_{1}, \hat{r}_{2}\right)
$$

and

$$
C_{\lambda_{1}, \lambda_{2}, \Lambda}\left(\nabla_{1}^{Y}, \nabla_{2}^{Y}\right)=\frac{b_{\lambda_{1}} b_{\lambda_{2}}}{r_{1} r_{2}} C_{\lambda_{1}, \lambda_{2}, \Lambda}\left(\hat{r}_{1}, \hat{r}_{2}\right),
$$

where

$$
b_{\lambda_{i}}=\left\{\begin{array}{l}
-l_{i} \text { if } \lambda_{i}=l_{i}+1 \\
l_{i}+1 \text { if } \lambda_{i}=l_{i}-1
\end{array}\right.
$$

for $i=1,2$. The above is valid for any vectorcoupled product $\left(r_{1}, \nabla_{2}^{Y}\right)_{Q}^{K}$ or $\left(\nabla_{1}^{Y}, \nabla_{2}^{Y}\right)_{Q}^{K}$ with $K=0,1$, 2. The radial factors in (27) and (28) are to be included in the $I_{\Lambda}\left(R^{\prime} R\right)$ radial integrals. As an example, the complete reduction to radial integrals for the operator $\hat{r}_{1} \times \nabla_{2}$ is

$$
I\left(\hat{r}_{1} \times \nabla_{2}\right)=\sum_{\lambda_{1}, \lambda_{2}, \Lambda} C_{\lambda_{1}, \lambda_{2}, \Lambda}\left(\hat{r}_{1} \times \hat{r}_{2}\right) I_{\Lambda}\left(R^{\prime} \Omega_{\lambda_{2}} R\right),
$$

with

$$
\begin{aligned}
C_{\Lambda}= & \frac{(-1)^{L^{\prime}-M^{\prime}}}{2(4 \pi)^{1 / 2}}\left(\begin{array}{ccc}
L^{\prime} & k & L \\
-M^{\prime} & q & M
\end{array}\right)(-1)^{l_{2}^{\prime}+L^{\prime}+L+k}\left(l_{1}, l_{1}^{\prime}, l_{2}, l_{2}^{\prime}, L, L^{\prime}\right)^{1 / 2} \\
& \times \sum_{\lambda}(\lambda, \Lambda, k)\left(\begin{array}{lll}
l_{1} & k & \lambda \\
0 & 0 & 0
\end{array}\right)\left(\begin{array}{lll}
l_{1}^{\prime} & \lambda & \Lambda \\
0 & 0 & 0
\end{array}\right)\left(\begin{array}{lll}
l_{2}^{\prime} & l_{2} & \Lambda \\
0 & 0 & 0
\end{array}\right)\left\{\begin{array}{lll}
L^{\prime} & \lambda & l_{2} \\
\Lambda & l_{2}^{\prime} & l_{1}^{\prime}
\end{array}\right\}\left\{\begin{array}{lll}
L^{\prime} & \lambda & l_{2} \\
l_{1} & L & k
\end{array}\right\} .
\end{aligned}
$$

The corresponding result for $Y_{k}^{\alpha}\left(\hat{\gamma}_{2}\right)$ is obtained by interchanging the subscripts 1 and 2 throughout and multiplying by $(-1)^{L+L^{\prime}+k}$. For the dipole operator, the only nonvanishing contributions come from $\lambda=l_{1} \pm 1$.

\section{Breit interaction}

The matrix elements of the Breit interaction ${ }^{10}$ are of considerable interest in atomic-structure calculations and have been worked out in detail

$$
\Omega_{\lambda_{2}}=\frac{\partial}{\partial r_{2}}+\frac{r_{1}}{r} \frac{\partial}{\partial r}+\frac{b \lambda_{2}}{r_{2}} .
$$

The Laplacian operator for particle 1 in our notation is

$$
\begin{aligned}
\nabla_{1}^{2}= & \frac{1}{r_{1}^{2}} \frac{\partial}{\partial r_{1}}\left(r_{1}^{2} \frac{\partial}{\partial r_{1}}\right)+\frac{1}{r^{2}} \frac{\partial}{\partial r}\left(r^{2} \frac{\partial}{\partial r}\right)-\frac{\left(l_{1}^{Y}\right)^{2}}{r_{1}^{2}} \\
& +\frac{2\left(r_{1}-r_{2} \cos \theta\right)}{r} \frac{\partial^{2}}{\partial r_{1} \partial r}-2\left(\nabla_{1}^{Y} \cdot \vec{r}_{2}\right) \frac{1}{r} \frac{\partial}{\partial r},
\end{aligned}
$$

where $\left(l_{1}^{Y}\right)^{2}$ acts only on the spherical harmonics. The corresponding result for $\nabla_{2}^{2}$ is obtained by interchanging the subscripts 1 and 2 .

\section{Multipole transition integrals}

Consider matrix elements of $Y_{k}^{q}\left(\hat{r}_{1}\right)$ $=\sqrt{4 \pi} T_{k, 0, k}^{q}\left(\hat{r}_{1}, \hat{r}_{2}\right)$. Since the $C_{\Lambda}$ vanish unless $\lambda_{2}=l_{2}$ in (14), we obtain

$$
\begin{aligned}
H_{2}=\frac{\alpha^{2}}{2}\{ & \frac{\left(2 r^{2} \cos \theta-r_{1} r_{2} \sin ^{2} \theta\right)}{r^{3}} \frac{\partial^{2}}{\partial r_{1} \partial r_{2}}-\frac{2}{r^{2}} \frac{\partial}{\partial r}\left(r \frac{\partial}{\partial r}\right)+\frac{\left(r_{2}^{2}-r_{1}^{2}-r^{2}\right)}{r_{1} r^{2}} \frac{\partial^{2}}{\partial r_{1} \partial r}+\frac{\left(r_{1}^{2}-r_{2}^{2}-r^{2}\right)}{r_{2} r^{2}} \frac{\partial^{2}}{\partial r_{2} \partial r} \\
& +\left(\overrightarrow{\mathrm{r}}_{1} \cdot \nabla_{2}^{Y}\right)\left[\frac{\left(r_{1}^{2}-r_{2}^{2}+3 r^{2}\right)}{2 r_{1} r^{3}} \frac{\partial}{\partial r_{1}}+\frac{2}{r^{2}} \frac{\partial}{\partial r}\right]+\left(\overrightarrow{\mathrm{r}}_{2} \cdot \nabla_{1}^{Y}\right)\left[\frac{\left(r_{2}^{2}-r_{1}^{2}+3 r^{2}\right)}{2 r_{2} r^{3}} \frac{\partial}{\partial r_{2}}+\frac{2}{r^{2}} \frac{\partial}{\partial r}\right] \\
& \left.+\left(\nabla_{1}^{Y} \cdot \nabla_{2}^{Y}\right) \frac{\left(r^{2}-r_{1} r_{2} \cos \theta\right)}{r^{3}}-\frac{\left(L^{2}-l_{1}^{2}-l_{2}^{2}\right)}{2 r^{3}}\right\} .
\end{aligned}
$$

As before, $\nabla_{1}^{Y}, \nabla_{2}^{Y}, \overrightarrow{\mathrm{I}}_{1}$, and $\overrightarrow{\mathrm{I}}_{2}$ act only on the spherical harmonic part of the wave function. The parts of (34) not containing $\nabla^{Y}$ factors are the same as those given by Schiff $e t$ al. ${ }^{1}$ while the rest is a generalization of their results to arbitrary angular by Schiff et al. ${ }^{1}$ for the special case of $1 s n p^{1,3} P$ states. Using the methods already developed here, the general reduction to radial integrals is straightforward for all the terms, except for the orbitorbit interaction given by

$H_{2}=\alpha^{2} / 2\left[(1 / r) \nabla_{1} \cdot \nabla_{2}+\left(1 / r^{3}\right) \overrightarrow{\mathrm{r}}_{12}\left(\overrightarrow{\mathrm{r}}_{12} \cdot \nabla_{1}\right) \nabla_{2}\right]$

in atomic units, where $\alpha$ is the fine-structure coristant and $\vec{r}_{12}=\vec{r}_{1}-\vec{r}_{2}$. This operator can also be reduced in general to radial integrals if (23a) and (23b) are first used to express $H_{2}$ in the form momentum. The angular coefficients for the latter parts are given by (27) and (28), while the former parts require only the overlap coefficients (20). Factors of $\cos \theta$ do not affect the angular integration since these are purely radial functions given 
by (9).

The spin-spin type interactions of the form

$$
H_{s s}=\alpha^{2} / r^{3}\left[\overrightarrow{\mathrm{s}}_{1} \cdot \overrightarrow{\mathrm{s}}_{2}-3\left(\overrightarrow{\mathrm{s}}_{1} \cdot \overrightarrow{\mathrm{r}}_{12}\right)\left(\overrightarrow{\mathrm{s}}_{2} \cdot \overrightarrow{\mathrm{r}}_{12}\right) / r^{2}\right]
$$

do not present any new difficulties if $H_{s s}$ is first written in the scalar product form ${ }^{11}$

$$
H_{s s}=\underline{S}^{(2)} \cdot \underline{L}^{(2)} \text {, }
$$

where $\underline{S}^{(2)}$ and $\underline{L}^{(2)}$ are irreducible tensors of rank 2 given by

$$
\begin{aligned}
& S_{m}^{(2)}=\left(\frac{2}{5} \pi\right)^{1 / 2}\left(\overrightarrow{\mathrm{S}}_{1} \cdot \nabla\right)\left(\overrightarrow{\mathrm{S}}_{2} \cdot \nabla\right) r^{2} Y_{2}^{m}(\hat{r}), \\
& L_{m}^{(2)}=\left(\frac{8}{5} \pi\right)^{1 / 2}\left(\alpha^{2} / r^{3}\right) Y_{2}^{m}\left(\hat{r}_{12}\right) .
\end{aligned}
$$

The angular coefficients for the matrix elements of $L_{m}^{(2)}$ in the uncoupled $\left(L, S, M_{L}, M_{S}\right)$ scheme can be found from the decomposition

$$
\begin{aligned}
Y_{2}^{m}\left(\hat{r}_{12}\right)= & \left(r_{1}^{2} / r^{2}\right) Y_{2}^{m}\left(\hat{r}_{1}\right)+\left(r_{2}^{2} / r^{2}\right) Y_{2}^{m}\left(\hat{r}_{2}\right) \\
& -2\left(\frac{10}{3} \pi\right)^{1 / 2} T_{112}^{m}\left(\hat{r}_{1}, \hat{r}_{2}\right) .
\end{aligned}
$$

The $C_{\mathrm{A}}$ for the first two terms are obtained either directly from (32) or from its interchange as discussed in Sec. III C. The $C_{\Lambda}$ for the last term are obtained from (14) and (15). The final result for a particular matrix element of $L_{m}^{(2)}$ in the uncoupled representation is

$$
\begin{aligned}
\left\langle l_{1}^{\prime} l_{2}^{\prime} L^{\prime} M^{\prime}\left|L_{m}^{(2)}\right| l_{1} l_{2} L M\right\rangle & \\
=\left(\frac{8}{5} \pi\right)^{1 / 2} \alpha^{2} \sum_{\Lambda} & \left\{C_{\Lambda}\left[Y_{2}^{m}\left(\hat{r}_{1}\right)\right]\left(\frac{R^{\prime} R r_{1}^{2}}{r^{5}}\right)\right. \\
+ & C_{\Lambda}\left[Y_{2}^{m}\left(\hat{r}_{2}\right)\right] I_{\Lambda}\left(\frac{R^{\prime} R r_{2}^{2}}{r^{5}}\right) \\
& \left.-2\left(\frac{10}{3} \pi\right)^{1 / 2} C_{\Lambda}\left(T_{112}^{m}\right) I_{\Lambda}\left(\frac{R^{\prime} R}{r^{3}}\right)\right\} .
\end{aligned}
$$

This determines the reduced matrix element of $L^{(2)}$ in

$$
\begin{aligned}
& \left\langle\gamma^{\prime} L S^{\prime} J^{\prime} M^{\prime}\left|\underline{L}^{(2)} \cdot \underline{S}^{(2)}\right| \gamma L S J M\right\rangle \\
& =(-1)^{L+S^{\prime}+J} \delta_{J^{\prime}, J} \delta_{M^{\prime}, M}\left\{\begin{array}{lll}
J & S^{\prime} & L^{\prime} \\
2 & L & S
\end{array}\right\} \\
& \times\left\langle\gamma^{\prime} L^{\prime}\left\|L^{(2)}\right\| \gamma L\right\rangle\left\langle S^{\prime}\left\|S^{(2)}\right\| S\right\rangle .
\end{aligned}
$$

A similar reduction can be written down immediately for the spin-orbit and spin-other-orbit interactions.

\section{RADIAL INTEGRAL RECURRENCE RELATIONS}

The results of Secs. I-III reduce the matrix elements for a wide variety of operators to sums of radial integrals (8), which we now write in the abbreviated form

$$
\begin{aligned}
I_{l}(g)=\int_{0}^{\infty} r_{1} d r_{1} \int_{0}^{\infty} r_{2} d r_{2} f\left(r_{1}, r_{2}\right) \\
\\
\quad \times \int_{\left|r_{1}-r_{2}\right|}^{r_{1}+r_{2}} r d r g(r) P_{l}(\cos \theta),
\end{aligned}
$$

where $f\left(r_{1}, r_{2}\right)$ and $g(r)$ are any functions for which the integral exists. A recurrence relation for the $I_{l}(g)$ is obtained by first considering the coefficients $R_{l}(g)$ in the Legendre polynomial expansion ${ }^{4}$

$$
g(r)=\sum_{L=0}^{\infty} R_{L}(g) P_{L}(\cos \theta)
$$

This is also an abbreviated notation since the $R_{L}(g)$ are functions of $r_{1}$ and $r_{2}$. Multiplying (43) by $P_{l}(\cos \theta) d \cos \theta$ and integrating from -1 to +1 yields

$$
R_{l}(g)=\frac{2 l+1}{2 r_{1} r_{2}} J_{l}(g)
$$

where

$$
J_{l}(g)=\int_{\left|r_{1}-r_{2}\right|}^{r_{1}+r_{2}} r d r g(r) P_{l}(\cos \theta) .
$$

In (45), the variable of integration has been changed to $r=\left(r_{1}^{2}+r_{2}^{2}-2 r_{1} r_{2} \cos \theta\right)^{1 / 2}$. A recurrence relation for the $J_{l}(g)$ is obtained by substituting

$$
P_{l}(x)=\left[P_{l+1}^{\prime}(x)-P_{l-1}^{\prime}(x)\right] /(2 l+1),
$$

and integrating by parts with the result

$$
J_{l+1}\left(g^{\prime} / r\right)=\frac{2 l+1}{r_{1} r_{2}} J_{l}(g)+J_{l-1}\left(g^{\prime} / r\right) .
$$

In each case, the prime means differentiation with respect to the argument. It is clear from the definitions (42) and (45) that the $I_{l}(g)$ satisfy the analogous recurrence relation

$$
I_{l+1}\left(g^{\prime} / r\right)=(2 l+1) I_{l}\left(r_{1}^{-1} r_{2}^{-1} g\right)+I_{l-1}\left(g^{\prime} / r\right)
$$

Equation (48) generates a wide variety of integral recurrence relations depending on the choice made for the function $g(r)$. For integrals containing only powers of $r$, the choice $g(r)=r^{c+2}$ yields

$I_{l+1}\left(r^{c}\right)=\frac{2 l+1}{c+2} I_{l}\left(r_{1}^{-1} r_{2}^{-1} r^{c+2}\right)+I_{l-1}\left(r^{c}\right), \quad c \neq-2$.

The special case with $l=1$ was employed in the calculations of Schwartz. ${ }^{12}$ Except for the case $c=-2$, the relation is valid whenever the integrals exist. It allows the efficient and rapid computation of all $I_{l}\left(r^{c}\right)$ from a smaller table of values of $I_{0}\left(\gamma_{1}^{a} \gamma_{2}^{b} r^{c}\right)$ than would be required for the direct evaluation of (42). Using (42) and (9), the values of $I_{1}\left(r_{1}^{a} \gamma_{2}^{b} r^{c}\right)$ needed to start the recurrence relation are

$$
\begin{aligned}
I_{1}\left(\gamma_{1}^{a} r_{2}^{b} r^{c}\right)=\frac{1}{2}[ & I_{0}\left(\gamma_{1}^{a+1} \gamma_{2}^{b-1} r^{c}\right)+I_{0}\left(\gamma_{1}^{a-1} r_{2}^{b+1} r^{c}\right) \\
& \left.-I_{0}\left(r_{1}^{a-1} r_{2}^{b-1} r^{c+2}\right)\right] .
\end{aligned}
$$


Equation (49) is indeterminate for $c=-2$, but the choice $g(r)=\ln r$ yields the well-defined equation

$$
I_{l+1}\left(r^{-2}\right)=(2 l+1) I_{l}\left(r_{1}^{-1} r_{2}^{-1} \ln r\right)+I_{l-1}\left(r^{-2}\right)
$$

to be used in place of (49) when $c=-2$. A recurrence relation for logarithmic integrals follows immediately from (48) with the choice $g(r)$ $=r^{c+2} \ln r-r^{c+2} /(c+2)$, resulting in

$$
\begin{aligned}
I_{l+1}\left(r^{c} \ln \gamma\right)= & \frac{(2 l+1)}{c+2}\left[I_{l}\left(r_{1}^{-1} r_{2}^{-1} r^{c+2} \ln r\right)\right. \\
& \left.-I_{l}\left(r_{1}^{-1} r_{2}^{-1} r^{c+2}\right) /(c+2)\right]+I_{l-1}\left(r^{c} \ln \gamma\right) .
\end{aligned}
$$

Using (9) to replace a factor of $r^{2}$ in the integrand of $I_{t}\left(r_{1}^{-1} r_{2}^{-1} r^{c+2} \ln \gamma\right)$, along with the recurrence relation for the Legendre polynomials, (52) becomes

$$
(c+2 l+4) I_{l+1}\left(r^{c} \ln r\right)
$$

$$
\begin{aligned}
& =(2 l+1) I_{l}\left[\left(r_{1}^{2}+r_{2}^{2}\right) r_{1}^{-1} r_{2}^{-1} r^{c} \ln r\right] \\
& -\frac{1}{(c+2)} I_{l}\left(r_{1}^{-1} r_{2}^{-1} r^{c+2}\right) \\
& +(c-2 l+2) I_{l-1}\left(r^{c} \ln r\right)
\end{aligned}
$$

The same technique applied to the term $I_{l}\left(r_{1}^{-1} r_{2}^{-1} r^{c+2}\right)$ in (50) yields the recurrence relation

$$
\begin{aligned}
(c+2 l+4) I_{l+1}\left(r^{c}\right)= & (2 l+1) I_{l}\left[\left(r_{1}^{2}+r_{2}^{2}\right) r_{1}^{-1} r_{2}^{-1} r^{c}\right] \\
& +(c-2 l+2) I_{l-1}\left(r^{c}\right) .
\end{aligned}
$$

Many other integral relations can be derived in the same way. The choice $g(r)=\exp \left(-r^{2}\right)$ leads to the particularly simple result

$$
-2 I_{l+1}\left(e^{-r^{2}}\right)=(2 l+1) I_{l}\left(r_{1}^{-1} r_{2}^{-1} e^{-r^{2}}\right)-2 I_{l-1}\left(e^{-r^{2}}\right) .
$$

The corresponding recurrence relations for the coefficients $R_{l}(g)$ in the Legendre polynomial expansion (43) can easily be derived. From (44) and (47), the general relation is

$$
\frac{R_{l+1}\left(g^{\prime} / r\right)}{(2 l+3)}=\frac{R_{l}(g)}{r_{1} r_{2}}+\frac{R_{l-1}\left(g^{\prime} / r\right)}{(2 l-1)}
$$

The choice $g(r)=r^{c+2}$ leads to the recurrence relation

$$
\frac{R_{l+1}\left(r^{c}\right)}{(2 l+3)}=\frac{R_{l}\left(r^{c+2}\right)}{(c+2) r_{1} r_{2}}+\frac{R_{l-1}\left(r^{c}\right)}{(2 l-1)}
$$

which is equivalent to Eq. (31c) of Sack $^{4}$ obtained by a more lengthy procedure.

Similarly, the analog of (54) is

$$
\begin{aligned}
\frac{(c+2 l+4)}{(2 l+3)} R_{l+1}\left(r^{c}\right)= & \frac{\left(r_{1}^{2}+r_{2}^{2}\right)}{r_{1} r_{2}} R_{l}\left(r^{c}\right) \\
& +\frac{(c-2 l+2)}{(2 l-1)} R_{l-1}\left(r^{c}\right)
\end{aligned}
$$

and the analog of (53) is

$$
\begin{aligned}
(c+2 l+4) & /(2 l+3) R_{l+1}\left(r^{c} \ln r\right) \\
= & 1 / r_{1} r_{2}\left[\left(r_{1}^{2}+r_{2}^{2}\right) R_{l}\left(r^{c} \ln r\right)-\frac{1}{(c+2)} R_{l}\left(r^{c+2}\right)\right] \\
& +\frac{(c-2 l+2)}{(2 l-1)} R_{l-1}\left(r^{c} \ln r\right)
\end{aligned}
$$

Sack did not derive an expression equivalent to (59), but (59) reduces to his equation (39) for the case $c=0$. The choice $g(r)=\exp \left(-\alpha r^{2}\right)$ yields

$-\frac{2 \alpha R_{l+1}\left(e^{-\alpha r^{2}}\right)}{2 l+3}=\frac{R_{l}\left(e^{-\alpha r^{2}}\right)}{r_{1} r_{2}}-\frac{2 \alpha R_{l-1}\left(e^{-\alpha r^{2}}\right)}{2 l-1}$

for the coefficients in the Legendre polynomial expansion of $\exp \left(-\alpha r^{2}\right)$. The above formula reproduces the first few terms given by Swiatecki.13 Other recurrence relations, including those given by Sack, can easily be obtained by using (9) to replace factors of $r^{2}$ in the integrand of (45), together with recurrence relations for the Legendre polynomials.

Most of the formulas in this paper have been checked for accuracy in a number of special cases. In particular, recurrence relations such as (48) and (51) have now been used in a large number of calculations with correlated variational wave functions, ${ }^{14}$ and have been found to be very stable against round-off error when used in the forward direction. Together with the angular integration formulas, the results make possible the construction of efficient general-purpose integration routines to handle most of the matrix elements encountered in two-electron problems.

\section{ACKNOWLEDGMENT}

Research support by the National Research Council of Canada is gratefully acknowledged.

\footnotetext{
${ }^{1}$ B. Schiff, H. Lifson, C. L. Peckeris, and P. Rabinowitz, Phys. Rev. 140, A1104 (1965).

${ }^{2}$ L. Hambro, Phys. Rev. A $\underline{5}, 2027$ (1972); $\underline{6}, 865$ (1972);

G. W. F. Drake, G. A. Victor, and A. Dalgarno, Phys.
}

Rev. 180, 25 (1969).

${ }^{3}$ J.-L. Calais and P.-O. Löwdin, J. Mol. Spectrosc. $\underline{8}$, 203 (1962).

${ }^{4}$ R. A. Sack, J. Math. Phys. $\underline{5}, 245$ (1964). 
${ }^{5} \mathrm{H}$. Margenau and G. M. Murphy. The Mathematics of Physics and Chemistry (Van Nostrand, Princeton, N. J. 1956), p. 383.

${ }^{6}$ D. M. Brink and G. R. Satchler, Angular Momentum (Clarendon, Oxford, 1968).

${ }^{7} \mathrm{~A}$. R. Edmonds, Angular Momentum in Quantum Mechanics (Princeton University, Princeton, N. J. 1960), p. 84.

${ }^{8}$ Ref. 6 , p. 133.

${ }^{9}$ Ref. 6 , p. 141.

${ }^{10} \mathrm{H}$. A. Bethe and E. E. Salpeter, Quantum Mechanics of
One-and Two-Electron Systems (Springer-Verlag, Berlin, 1957) p. 181.

${ }^{11}$ Ref. 7. pp. 73 and 118.

${ }^{12}$ C. Schwartz, Phys. Rev. 134, A1181 (1964).

${ }^{13}$ W. J. Swiatecki, Proc. Roy. Soc. Lond. A 205, 238 (1951).

${ }^{14}$ See, for example, G. W. F. Drake, Phys. Rev. A $\underline{5}$, 614 (1972); G. W. F. Drake and A. Dalgarno, ibid.A 1 1325 (1970); G. W. F. Drake and A. Dalgarno, AstroPhys. J. 157, 459 (1969). 